\title{
Le spécialiste de la documentation et l'Année mondiale des communications
}

\section{The Specialist in Information and the World Year of Communications \\ El especialista de la documentación y el Año Mundial de las Comunicaciones}

Jean de Bonville

Volume 29, numéro 3, juillet-septembre 1983

URI : https://id.erudit.org/iderudit/1053619ar

DOI : https://doi.org/10.7202/1053619ar

Aller au sommaire du numéro

Éditeur(s)

Association pour l'avancement des sciences et des techniques de la documentation (ASTED)

ISSN

0315-2340 (imprimé)

2291-8949 (numérique)

Découvrir la revue

Citer cet article

de Bonville, J. (1983). Le spécialiste de la documentation et l'Année mondiale des communications. Documentation et bibliothèques, 29(3), 93-97.

https://doi.org/10.7202/1053619ar
Résumé de l'article

Réflexion autour de trois aspects suggérés par l'Année mondiale des communications : la fonction de communication inhérente à l'activité même des services documentaires, la transformation de l'environnement technologique du transfert de l'information par les médias et, enfin, la définition de l'activité professionnelle du spécialiste de la documentation à partir des concepts d'information et de communication.
Tous droits réservés (c) Association pour l'avancement des sciences et des techniques de la documentation (ASTED), 1983
Ce document est protégé par la loi sur le droit d'auteur. L'utilisation des services d'Érudit (y compris la reproduction) est assujettie à sa politique d'utilisation que vous pouvez consulter en ligne. 


\title{
Le spécialiste de la documentation et l'Année mondiale des communications
}

\author{
Jean de Bonville* \\ Université Laval \\ Québec
}

Réflexion autour de trois aspects suggérés par l'Année mondiale des communications: la fonction de communication inhérente à l'activité même des services documentaires, la transformation de l'environnement technologique du transfert de l'information par les médias et, enfin, la définition de l'activité professionnelle du spécialiste de la documentation à partir des concepts d'information et de communication.

\section{The Specialist in Information and the World Year of Communications}

Thoughts centered on three points of view prompted by the World Year of Communications: the communicating function inherent in the activities of information services; the changes in technological means for imparting information; and, lastly, the professional activity of the specialist in information as understood from the concepts of information and communication.
El especialista de la documentación y el Año Mundial de las Comunicaciones

Reflexión sobre tres aspectos sugeridos por el Año Mundial de las Comunicaciones: la función de comunicación inherente a la actividad misma de los servicios documentales, la transformación delmedio ambiente technológico de la transferencia de información por los medios y, finalmente, la definición de la actividad profesional del especialista de la documentación a partir de los conceptos de información y de comunicación.
Les suggestions des organismes internationaux sont une source d'inspiration à laquelle puisent abondamment les associations professionnelles pour établir la thématique de leurs congrès. Cette convention, qui peut manquer d'originalité au goût de certains, a l'avantage de susciter la réflexion sur des aspects neufs ou de stimuler l'intérêt pour des sujets déjà anciens. En exploitant les suggestions des institutions des Nations Unies. on a donc proposé aux bibliothécaires du monde entier, ces dernières années, de réfléchir à la relation qu'établissent avec le monde du livre et les bibliothèques, les personnes handicapées. les enfants, les femmes...

En 1983. I'ASTED a choisi de faire porter son congrès sur le thème de la communication répondant au souhait de l'Union internationale

\footnotetext{
- L'auteur est conseiller à la documentation en communication
}

des télécommunications, responsable de l'organisation de l'Année mondiale des communications. C'est l'occasion pour les professionnels de la documentation de se pencher sur les problèmes de communication de leurs activités comme précédemment ils l'ont fait à propos des enfants, des personnes handicapées, etc. Certes le parallèle est justifié. Cependant, en abordant le thème des communications, ils touchent à un concept central de leur activité professionnelle. En fait, ils atteignent le coeur et le nerf même de leur fonction sociale.

Affirmation gratuite pour les uns, cliché pour les autres, cette proposition n'en demeure pas moins stimulante si on consent à prospecter ses ramifications. Je proposerai trois réflexions, l'une sur les fonctions de la communication, une deuxième sur les médias et, la dernière, sur les concepts même d'information et de communication. Ces réflexions nous amèneront du nerf, 
canal des impulsions, au coeur même de l'activité professionnelle du spécialiste de la documentation.

La fonction communication est essentielle à la réalisation optimale des objectifs d'un service documentaire. Elle est une condition de l'adéquation des prestations de services aux besoins des usagers. On a reconnu son importance même dans les approches traditionnelles de la bibliothèque et de la documentation. Les concepts de publicité et de relations publiques sont déjà familiers à tous. La communication joue un grand rôle, dans le fonctionnement interne de la bibliothèque, comme de toute organisation, et dans la relation de la bibliothèque avec son environnement.

Le fonctionnement quotidien d'un service documentaire suppose un système de communication assurant la circulation des informations sur le service et permettant aux membres de ce service de participer étroitement à ses activités et d'épouser ses objectifs. La relation avec la clientèle repose sur un ensemble de signes qui constituent autant de langages: langage de l'affichage et des signes permettant le repérage des services et des ressources, langage documentaire permettant le repérage de l'information. rhétorique de la négociation des besoins documentaires avec les clients du service. Une politique efficace de communication interne relève de plusieurs approches distinctes: signalétique, science de l'information, psychosociologie, psychologie, etc.

La communication avec son environnement s'est souvent exprimée, pour le service documentaire, d'une manière unidirectionnelle: effort de promotion et de publicité des services et des ressources. Or, une communication efficace. même dans le but d'une démarche publicitaire. doit prendre en compte l'interaction des besoins et des demandes d'une part et des services d'autre part. Cette interaction entraîne souvent une dialectique favorable à l'élaboration d'une stratégie judicieuse et efficace de communication externe.

Pour promouvoir l'utilisation des ressources humaines et documentaires de leur bibliothèque. les responsables insistent souvent sur le volume de la documentation disponible, sur la variété des supports d'information. Mais ces renseignements demeurent souvent impuissants à persuader les utilisateurs potentiels de la bibliothèque de se prévaloir de ses services. Les techniques de communication que constituent la publicité et les relations publiques ont permis la mise au point, avec l'aide des différentes sciences sociales, un ensemble d'instruments méthodologiques aujourd'hui nécessaires à une relation efficace de l'organisation avec son environnement. Qu'on puisse contester la finalité des activités auxquelles ces instruments sont appliqués n'entame pas leur valeur heuristique. Au contraire, leur utilisation efficace à des fins parfois contestables démontre leur puissance.

Ces techniques permettent une appréhension fine de phénomènes complexes: nature et composition de la clientèle, attentes, motivations. besoins. On entre ici sur la scène de la communication symbolique où les images et les stéréotypes jouent un rôle de premier plan. Image de soi d'abord: quelle opinion le client se fait-il de ses besoins d'information et de documentation? Quelle est, selon lui, son aptitude à solutionner ses problèmes d'information? Les demandes exprimées par l'utilisateur des services documentaires masquent souvent les véritables besoins. Par exemple, une question sur une vedettematière peut exprimer, implicitement, un besoin de formation sur les instruments de repérage du service documentaire. La sous-utilisation systématique de tel type d'instruments peut être un signe de leur méconnaissance par les utilisateurs ou de l'incompétence de ceux-ci à les utiliser.

Image de l'information et de la documentation aussi. La documentation est-elle une ressource essentielle à la créativité, un stimulant heuristique ou simplement la source ponctuelle et occasionnelle de renseignements utilitaires? Ou encore. un fardeau à porter pour respecter les prescriptions du style "académique» : citations, notes infrapaginales, bibliographies.

Image du service documentaire et de ses ressources. L'image que se font les utilisateurs du personnel d'un service documentaire influence lourdement la nature des problèmes et des besoins exposés par eux. Selon que le personnel au service des utilisateurs est composé de commis, de bibliotechniciens, de bibliothécaires ou de spécialistes, les sujets abordés par les clients et la nature des prestations attendues peuvent changer. Le besoin exprimé par les utilisateurs est en partie déterminé par la nature appréhendée des ressources humaines. Ils s'abstiendront de soumettre des besoins complexes d'information et de s'ouvrir sur la problématique même de la recherche documentaire s'ils perçoivent leur interlocuteur comme incapable de participer efficacement à la solution de ces problèmes. La sous-utilisation de certains services ou de certaines ressources est souvent attribuable à l'ignorance de leur existence même, à la méconnaissance de leur nature ou encore. s'agissant des documents eux-mêmes, à l'inaptitude des langages et systèmes documentaires à rendre compte adéquatement des informations qu'ils contiennent.

En résumé, la fonction de communication d'un service documentaire doit s'articuler dans des politiques de communication interne et externe. Elle doit puiser dans l'outillage méthodologique offert par les sciences humaines et sociales. Elle doit s'étayer de données quantitatives sur l'offre 
et la demande de services mais, tout autant, sur une observation précise des perceptions et attitudes des utilisateurs

La fonction de communication participe de la nature même des services documentaires et, à cet égard, son intérêt n'obéit pas à des préoccupations conjoncturelles. L'Année mondiale des communications, décrétée pour 1983, témoigne au contraire d'une évolution significative qui touche particulièrememt les médias. Depuis quelques décennies, et de plus en plus, ceux-ci pénètrent nos vies, transforment habitudes de travail et pratiques sociales, modèlent visions du monde et attitudes. Les prochaines années ne seront pas moins fertiles en changements technologiques que ne l'a été le dernier tiers de siècle. La tendance de ces changements s'affirme clairement. Des moyens de diffusion très puissants, appuyés par les capacités de sélection et de traitement de l'informatique, font passer les médias de l'âge de la «masse » à ceux du groupe et de l'individu. Multiplication des canaux de télévision, télévision en direct par satellite, fibres optiques, laser, communication interactive. vidéotex, courrier électronique, etc.

Cet arsenal, comme tiré d'une boîte de pandore par les gourous de la communication, n'a cependant rien de terrifiant. II suffit de l'apprivoiser. En fait, pour les spécialistes de la documentation, c'est une exigence. S'ils se tiennent en retrait de ces changements technologiques, ils se condamnent à l'isolement et, à terme, à l'inutilité. Dressons, à grands traits, un scénario que la technique rend possible dès maintenant mais qui ne se dessinera que progressivement à mesure que les résistances sociales, psychologiques et économiques s'effaceront. Les informations sont enregistrées au moment même de leur production dans de vastes mémoires (bandes et disques magnétiques. vidéodisques). Chaque citoyen peut avoir accès. de chez lui, à cette mémoire grâce à un terminal interactif relié à la mémoire par fibres optiques. S'il s'agit de données ponctuelles, il en prend connaissance sur son écran domestique. Si l'information recherchée occupe trop d'espace, il peut commander son édition sur papier grâce à l'imprimante au laser couplée à son écran. Les données et les informations ainsi requises ont été traitées au moment de leur stockage. Pour les informations contenues sur un support papier. car on conserve livres et périodiques anciens. lecteur optique et transmission par fac-similé permettent un accès rapide au document. Certes. il y a toujours des librairies et des bibliothèques. Elles servent essentiellement à satisfaire les besoins de loisirs culturels et de divertissement littéraire. Elles répondent au plaisir encore vif qu'on a de tenir un livre dans ses mains et de s'adonner à la lecture sans contrainte de lieu ni d'environnement technologique. Les bibliothèques ont en fait un statut quiles apparente au musée. Elles sont le lieu d'expériences culturelles recherchées. Mais pour satisfaire les besoins quotidiens d'information et de données, elles ont cédé la place à d'autres institutions, producteurs de banques et bases de données, éditeurs de progiciels, didacticiels et autres-ciels qui diffusent selon la demande les informations qu'ils créent. La télécommunication a aboli la nécessité d'aller vers le document: l'informatique permet un tri judicieux de l'information: des supports miniaturisés, à mémoire gigantesque et d'exploitation aisée, réduisent le volume nécessaire à l'enregistrement des données.

Les bibliothécaires auront tôt fait d'exorciser cette vision apocalyptique en la rangeant au rayon des chimères, utopies et autres rêveries. Sans y prendre garde, ils céderont à un réflexe de conservation inefficace et même dangereux. Quoi qu'on veuille, comme l'affirme F.W. Lancaster, "The paperless society is rapidly approaching»!

Le Petit Robert, en marquant l'ouverture sémantique du concept de bibliothèque, trace la voie de l'adaptation. A la suite de l'acception traditionnelle ("Salle, édifice où sont classés des livres, pour la lecture»), il ajoute, en bout de liste. une acception compatible avec le scénario brossé plus haut: "collection de supports d'information».

Dans la société de l'information, si les bibliothèques et les bibliothécaires sont marginalisés. ils en seront les responsables. Car ils ont en main tous les éléments pour entrer de plein pied dans la société de l'information. En fait, il leur faut s'approprier les nouveaux médias plutôt que de s'en aliéner. Ils doivent, pour le faire, comprendre la spécificité des changements technologiques qui toucheront l'information durant les prochaines décennies.

Depuis des siècles, le support et le moyen d'enregistrement de l'information sont demeurés les mêmes : feuilles de papier reliées ou brochées en livres ou fascicules de périodiques contenant des messages exprimés en caractères alphabétiques et numériques. Les moyens de repérer ces messages se sont constitués grâce à une opération intellectuelle d'abstraction qui a évolué et s'est raffinée: description bibliographique, catalogage, analyse, indexation. Les supports des renseignements permettant de repérer les messages se sont aussi modifiés: listes, fiches cartonnées. microfiches et maintenant disques magnétiques. Les transformations qui ont affecté les bibliothèques jusqu'à maintenant portaient sur les supports de l'information secondaire, c'est-àdire le catalogue bibliographique lui-même. Même les bases de données, considérées comme la fine pointe du développement en matière bibliographique, ne concernent qu'une infime

1 F.W. Lancaster, "Whither libraries? or wither libraries" College and Research Libraries. Sept. 1979, 357 
partie de l'information, à savoir l'information secondaire. Si elles influencent les modes d'accès à la description des documents, elles laissent pratiquement inchangée la relation au document lui-même, support de l'information primaire.

Au contraire, les changements technologiques à venir touchent à la fois l'accès à l'information secondaire et les supports de l'information primaire elle-même. L'accès à l'information secondaire, c'est-à-dire aux catalogues et aux bases de données bibliographiques, se libéralise. La consultation de ces sources d'information, qui se fait aujourd'hui en bibliothèque, se décentralise. On prend connaissance du signalement des sources d'information à la maison. Mais le changement le plus radical concerne l'appropriation de l'information primaire elle-même. désormais possible sans l'utilisation du support papier et sans déplacement vers un lieu physique et architectural appelé bibliothèque. Les médias ont réduit progressivement la distance entre l'information et son utilisateur jusqu'à l'abolir et, paradoxalement, c'est la bibliothèque qui vient au lecteur plutôt que le lecteur à la bibliothèque.

Ce scénario, que les prochaines décennies confirmeront, amène le bibliothécaire et les spécialistes de la documentation à reconsidérer l'objet spécifique de leur activité et les deux maitres mots pour les aider sont information et communication.

Le thème du congrès de I'ASTED en 1983 se formule ainsi tel un slogan: "Au delà de l'information: la communication». Comme tous les slogans, cet énoncé, pour mettre en valeur un aspect, introduit dans la réalité qu'il exprime une légère distorsion. La définition des concepts est. dès le départ, essentielle à la compréhension.

Information et communication sont deux termes polysémiques. Aux acceptations courantes de renseignement et de nouvelles se sont ajoutées des acceptions, tirées du domaine scientifique. qui ont approfondi le champ sémantique du concept d'information. Le Petit Robert retient une de ces acceptions introduite par la théorie mathématique de l'information: "mesure de la densité de renseignements contenus dans un message (pour un nombre de signes donnés)". Ailleurs, Abraham Moles formule autrement la même définition: l'information est "la mesure de la réduction de l'incertitude qu'il peut y avoir au sujet de l'état d'une partie de l'univers (ce qui se passe au lieu émetteur) par l'intermédiaire d'un message "2. Dans ce contexte, information n'est pas une valeur invariable et universelle; elle est au contraire une mesure qui peut varier, pour un même message, selon les interlocuteurs différents ou, selon les messages, pour un même inter- locuteur. L'information mesure l'utilité d'un message pour un interlocuteur donné. Autrement dit, elle mesure l'efficacité de la relation établie entre deux interlocuteurs. L'information n'est donc pas le message lui-même. ni encore moins le support sur lequel il est enregistré mais la part de renseignements nouveaux contenue dans le message et qui réduit l'incertitude de l'interlocuteur R (récepteur) à propos du sujet dont il traite avec l'interlocuteur $E$ (émetteur). L'énoncé "Beau soleil aujourd'hui». échangé en guise de politesse, permet d'établir une communication sans échanger d'information. La communication peut donc s' instaurer à d'autres fins que l'échange d'information. La relation s'établit souvent pour satisfaire des besoins affectifs ou sociaux.

D'évidence, les spécialistes de la documentation s'intéressent à la communication pour l'information qu'elle permet d'échanger. Dans ce contexte, on comprend qu'en tentant d'insister sur la relation de communication, le titre du congrès risque de faire oublier que cette communication, pour les spécialistes de la documentation, est au service de l'information. En fait, le slogan approprié pourrait être «l'information par la communication». Cet énoncé a l'avantage de souligner la dynamique essentielle de l'information. II n'y a pas d'information sans communication de données, de renseignements. de messages qui, pour le spécialiste de la documentation, sont enregistrés dans des documents.

Sans jeux de mots et sans effort sémantique. on peut donc affirmer que le bibliothécaire et le documentaliste sont des spécialistes de l'information et de la communication. Ils ne sont cependant pas les seuls à revendiquer ce statut. Le journaliste, le relationniste, le professeur, le juriste peuvent aussi se parer de ces titres. Leur prétention n'est pas moins fondée. En fait, les spécialistes de l'information et de la communication s'intéressent à des facettes ou à des aspects de l'information et de la communication. Bibliothécaires et documentalistes sont des agents actifs dans un type particulier de relation que j'ai déjà appelée la communication anonyme différée ${ }^{3}$. Dans ce type de communication, les interlocuteurs $E$ (émetteur) et $R$ (récepteur) ne se connaissent pas personnellement. Par exemple. l'auteur d'un livre sur la pollution des lacs ne connait pas tous les lecteurs de son livre et les lecteurs potentiels de ce livre ne connaissent pas non plus l'auteur. La communication est donc impersonnelle ou, pour employer un mot de Jean Lohisse, anonyme. La communication est différée parce que la relation entre les interlocuteurs s'établit en deux temps distincts, le temps 
d'émission et le temps de réception. Ces deux temps sont parfois très éloignés l'un de l'autre.

La responsabilité des spécialistes de la documentation est donc de solutionner les problèmes engendrés par la nature même de la communication anonyme différée : entropie engendrée par la multitude des émetteurs (auteurs) et des récepteurs (lecteurs), polysémie créée par la multiplicité des langages et messages, etc. Le rôle du spécialiste de la documentation est de concevoir et d'animer des services d'information capables de permettre une relation optimale entre interlocuteurs inconnus les uns des autres et souvent éloignés dans le temps et dans l'espace.

Bibliothécaires et documentalistes sont des spécialistes de l'information. Mais à la différence de l'historien et du juriste, spécialistes de l'information juridique ou historique, ils ne s'intéressent aux données, renseignements et messages, que dans la mesure où ils contiennent de l'information, quelle qu'elle soit. lls sont des spécialistes de l'information en tant que telle. En cela, ils sont proches parents du journaliste. Lui aussi établit une communication anonyme entre des interlocuteurs $E$ (sources d'information) et $R$ (auditoire). Lui aussi s'intéresse à toute information quelle qu'elle soit. Cependant, il intervient dans une communication non pas différée mais "simultanée». Il saisit le fait sur le vif et le communique au plut tôt. Spécialistes de la documentation et journalistes oeuvrent à la mise en valeur de l'information. Les uns s'attachent à la mise en valeur de la pertinence immédiate et circonstantielle de l'information: les autres s'intéressent à la mise en valeur de la pertinence permanente et universelle de l'information.

On voit, par cet exercice, que ce n'est pas en affadissant le sens des mots que bibliothécaires et documentalistes peuvent se dire spécialistes de l'information et de la communication. Ils le sont pleinement et au sens propre des termes bien qu'ils partagent avec d'autres cette responsabilité.

Mais à quoi bon, objectera-t-on, tant discourir sur les mots? Quelle prise ont-ils sur la réalité et quel changement, autre que lexical, de telles réflexions peuvent-elles provoquer? Evidemment, si les mots n'ont d'utilité que rhétorique et si la définition de l'activité professionnelle ne sert qu'à occuper le temps des congrès, force est de convenir qu'il est futile de s'attarder à circonscrire des concepts. Au contraire, si le langage exprime une réalité et s'il est un instrument participant à son évolution, la réflexion n'est pas vaine. Poser d'une part les concepts de conservation et de documentation ou leur préférer ceux d'information et de communication, c'est fixer des objectifs, dresser des programmes, établir des politiques.

L'Année mondiale des communications est le moment privilégié pour les bibliothécaires et les documentalistes de prendre acte de leur participation sans équivoque à la communication de l'information. Ils ne sont pas les invités de l'Année mondiale: ils doivent prendre place parmi les artisans de la fête. 